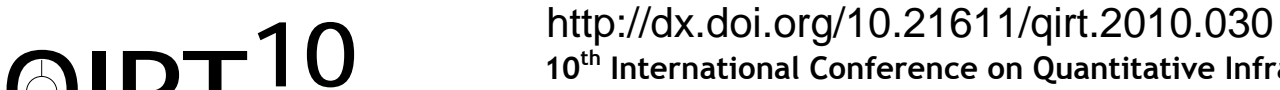 \\ $10^{\text {th }}$ International Conference on Quantitative InfraRed Thermography \\ July 27-30, 2010, Québec (Canada)
}

\section{Heat transfer enhancement with ribs in natural convection}

\author{
by G. Cardone* and M. Panelli**
}

\begin{abstract}
*DIAS, University of Naples “Federico II”, Piazzale Tecchio 80, 80125,Naples, Italy, gcardone@unina.it
**DIAS, University of Naples “Federico II", via Claudio, 80125,Naples, Italy, mario.panelli@unina.it
\end{abstract}

\begin{abstract}
Natural convection heat transfer is of a great interest in a lot of industrial applications: nuclear technologies, electronic circuit board cooling and many other fields. Only few studies have been carried out to determine the effect of surface roughness or shape on natural convective laminar flow. In this paper the effect of periodic patterns of protrusions (ribs) on the freeconvection heat transfer in a vertical plate, with uniform heat flux rate boundary condition, is experimentally investigated. The convective fluid is air. Heat transfer measurements are performed by using the infrared thermography and the heated thin foil technique.
\end{abstract}

\section{Introduction}

Natural convection heat transfer occurs in many engineering applications where heating and cooling is required. In particular, roughness elements or fins enhancing natural convection are studied both experimentally and numerically.

Bhavnani and Bergles [1] used an interferometric technique to determine local heat transfer coefficients for surfaces with repeated ribs and steps. The ribbed surfaces tested had square cross-sectioned ribs of height equal to 6.35 and 3.18 $\mathrm{mm}$, spaced at intervals of $25.4 \mathrm{~mm}$. The stepped test sections had steps with a constant height of $1.59 \mathrm{~mm}$ at spacings of 12.7, 25.4, and $50.8 \mathrm{~mm}$. The vertical plate $(127 \times 178 \mathrm{~mm}, 6.35 \mathrm{~mm}$ thick) made by aluminum (as ribs) had constant wall temperature $\left(48 \div 75^{\circ} \mathrm{C}\right)$. The flow was laminar. The maximum increase in average heat transfer coefficient was $23.2 \%$ with a step pitch to-height ratio of 16 . The study indicated the presence of an optimum step pitch-to-height ratio while all of the ribbed surfaces resulted in degraded heat transfer performance.

Aydin [2] studied natural convection of air over a constant heat-flux $\left(76 \div 88 \mathrm{~W} / \mathrm{m}^{2}\right)$ heated plate experimentally for the case of rib elements located in a laminar boundary layer. The aluminum plate had a width of $0.2 \mathrm{~m}$, a height of $1.21 \mathrm{~m}$. Two raws $(0.20 \mathrm{~m}$ long) of square ribs $(1 \mathrm{~cm}$ of edge) were mounted on the plate, $0.31 \mathrm{~m}$ far from plate's leading edge. The side to side spacing between ribs was $3.5 \mathrm{~cm}$ and $5 \mathrm{~cm}$. Aluminum and plexiglas were chosen as rib materials. Temperature measurements were performed using the IR thermography technique. It was shown that the ribs have not much influence on the local heat transfer coefficient (less than 10\%), based on an equal area of the flat plate.

Tanda [3] performed experiments to determine heat transfer data for the natural convective flow of air in vertical aluminum channels with one surface roughened by transverse square ribs $(4.85 \mathrm{~mm}$ high with $35 \mathrm{~mm}$ of pitch) and the opposite surface smooth. The plate without ribs was $175 \mathrm{~mm}$ height and $12 \mathrm{~mm}$ thick. Uniform wall temperature conditions were imposed on the ribbed side, while the smooth side remained unheated. The majority of tests were conducted by imposing a mean temperature difference between the heated plate and the ambient air of $45 \mathrm{~K}$, giving a Rayleigh number (based on the channel height as characteristic length) of 1.8.10\%. Additional experiments were carried out in vertical channels without ribs, under the same channel geometry and thermal conditions. A Schlieren optical technique was used to reconstruct the thermal field and to obtain distributions of heat transfer coefficients. The presence of ribs was found to alter heat transfer considerably, causing thermally inactive regions just upstream and downstream of each protrusion. Consequently, the heat transfer performance of the ribbed channel turned out to be lower than that of the corresponding smooth channel.

A liquid crystal based experimental investigation of the heat transfer enhancement supplied by ribs on a vertical plate $(304 \mathrm{~mm}$ high and $150 \mathrm{~mm}$ wide), has been presented by Onbasioglu [4]. Since the ribs were adiabatic, they did not work as extended heat transfer surfaces but by redirecting the flow. Two ribs with lengths of $67 \%$ of the heater width were placed perpendicular to the heater plate for each experimental case and were staggered on the plate. The experiment were performed varying the ribs pitch $(10,20,30,40 \mathrm{~mm})$ and considering five different angles of inclination $\left(0,10,20,30,45^{\circ}\right)$. The results indicated that ribs provide significant improvements in heat transfer, for the vertical plates.

The bibliographical research has shown that no periodic pattern of single or staggered raw of ribs were investigated. The aim of the paper is to fill this gap and to demonstrate that the use of periodic pattern of protrusion can enhance heat transfer of vertical plate, with uniform heat flux, in free laminar convective flow. The experimental analysis will be performed with IR thermography and the heated thin foil sensor [5]. 


\section{Experimental Apparatus}

The used experimental apparatus (see sketch in fig. 1) is a vertical stainless steel foil (245mm high, $960 \mathrm{~mm}$ wide, $40 \mu \mathrm{m}$ thick), coated on one side with a thin layer of high emissivity paint (0.95 in the range of temperature: [15-100] $\left.{ }^{\circ} \mathrm{C}\right)$ that is electrically heated obtaining an uniform boundary heat flux $(80 \div 270 \mathrm{~W} / \mathrm{m} 2)$. A suitable stiffening tool is also employed to assure the foil surface flatness.
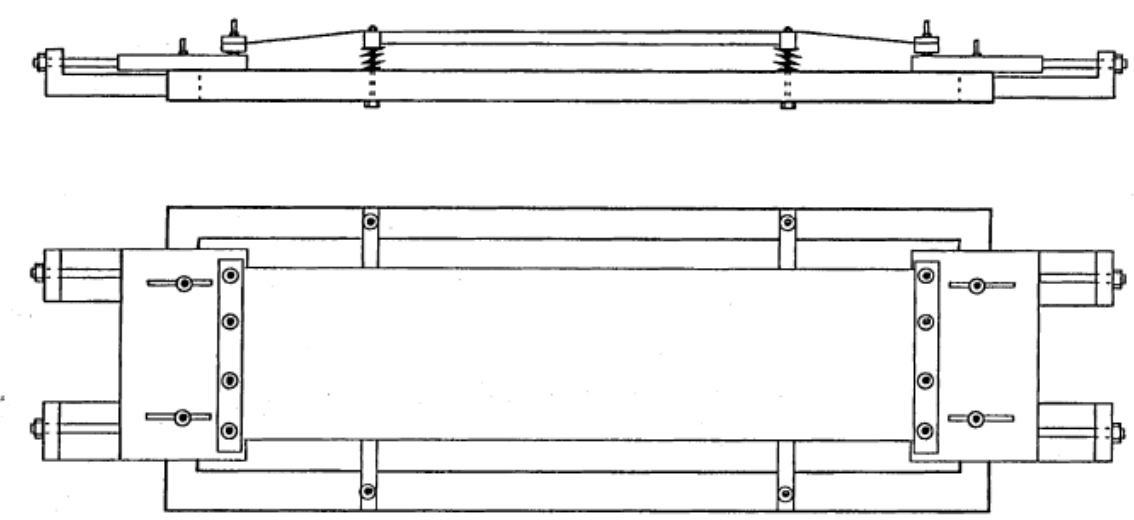

Fig. 1. Sketch of experimental apparatus.

Protuberances are obtained with right-angled magnetic parallelepiped elements $(4 \mathrm{~mm} \times 11 \mathrm{~mm} \times 44 \mathrm{~mm})$ that were place with different periodic patterns symmetrically on both surface of the vertical plate. The contact area between the magnets and the plate were electrically insulated using insulating tape. The use of magnet allows to test a lot of and also uncommon configurations in a little time, being the phase of ribs' positioning very fast.

The schematic representations of tested periodic patterns are shown in fig. 2 . The first class of measurements are performed on a single row of pattern of ribs (fig. 2a) considering two possible distances from leading edge of plate $(d=1 / 3 \mathrm{H}$ or $2 / 3 \mathrm{H}$ ) and four possible angles of inclination respect to the leading edge line $\left(\alpha=0,30,45,60^{\circ}\right)$; the pitch of ribs is $2 a$ where $a=L \cos (\alpha)$ and $L$ is ribs' length.

The second field of experiment has the aim to better understand the interaction of two staggered rows of protuberances (fig. 2b); the first raw of ribs, placed at $d=0.375 \mathrm{H}$, was inclined alternatively of $\alpha= \pm 45^{\circ}$ while the second row was placed at $d=2 / 3 \mathrm{H}$ with $\alpha=+45^{\circ}$.

The last one is the " $\mathrm{V}$ " type configuration (fig. 2c): two perpendicular ribs are inclined of $\alpha=+45^{\circ}$ forming " $\mathrm{v}$ " letter. The "V" type ribs are located at $d=1 / 3 \mathrm{H}$ and $2 / 3 \mathrm{H}$ with a pitch equal to $2.5 \mathrm{a}$.

FLIR SC6000 camera working the $8-12 \mu \mathrm{m}$ band is employed to measure the foil surface temperature. Heat transfer coefficients are computed from temperature map using, the heated thin foil technique [5], in particular:

$$
h=\frac{\dot{q}_{j}-\dot{q}_{r 1}-\dot{q}_{r 2}}{2\left(T_{w}-T_{i n f}\right)}
$$

Where $T_{w}$ is the wall temperature, $T_{\text {inf }}$ is the ambient temperature, $q_{j}$ is the Joule heating, $q_{r 1}$ and $q_{r 1}$ are the radiative heat fluxes from the front and back surfaces. The last ones may be directly evaluated by the Stefan Boltzmann's law considering that the different emissivity of the two sides (black side of plate has $\varepsilon_{1}=0.95$, and silver side has $\varepsilon_{2}=0.15$ ).

In natural convection flow, when the wall emissivity is high, the radiative heat flux is of the same order of magnitude (up to about one third) of the convective one. Measured heat transfer distribution are presented in non-dimensional form by means of the local Nusselt number $N u=h y / k$ where $y$ is the distance from leading edge and $k$ is the thermal conductivity coefficient of air evaluated at film temperature.

The experimental apparatus were located in a closed room in order to prevent any external airstream interfering with the free convection phenomena and a uniform boundary heat flux of $147 \mathrm{~W} / \mathrm{m}^{2}$ was established on the plate for all measurements. 


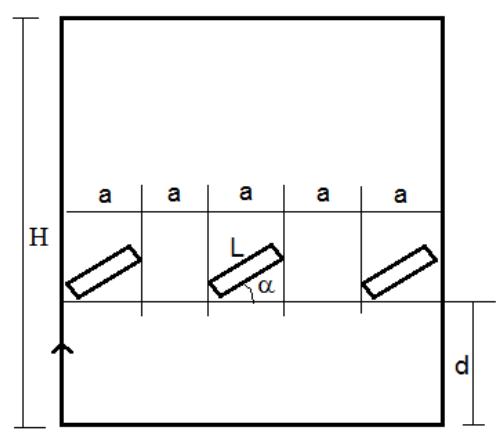

(a)

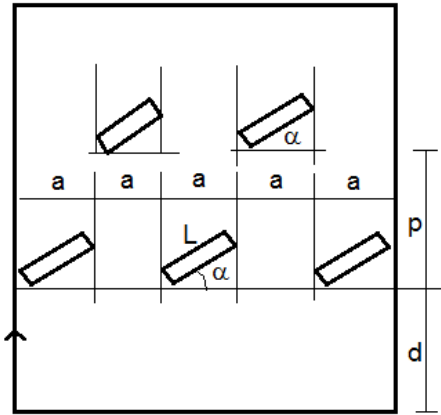

(b)

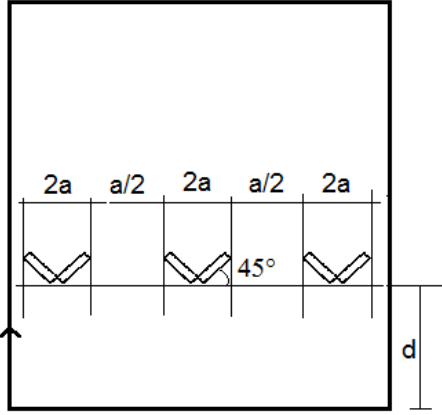

(c)

Fig. 3 Periodic patterns: (a) Single row; (b) two staggered rows; (c) " V "type.

\section{Results}

In order to validate the experimental methodology measurements, the Nusselt number on the flat plate without protrusion is compared with the classical correlation proposed by Sparrow [6] $N u^{\star}=0.6\left(G r^{\star} P r\right)^{0.2}=0.6\left(R a^{\star}\right)^{0.2}$ where $^{0} r^{*}$ is the modified Grashof number, $P r$ is the Prandtl number and $R a^{*}=G r^{\star} P r$ is the Rayleigh number. The modified $G r^{*}$ is defined as $G r^{*}=q_{c} \beta g y^{4} / k v^{2}$ where: $q_{c}$ is the wall convective flux, $\beta$ is the volumetric coefficient of thermal expansion, $g$ is the gravity constant and $v$ is the kinematic viscosity.

The experimental data for the vertical flat plate, reported in figure 3 , show good agreements with correlation in a wide range of Rayleigh number.

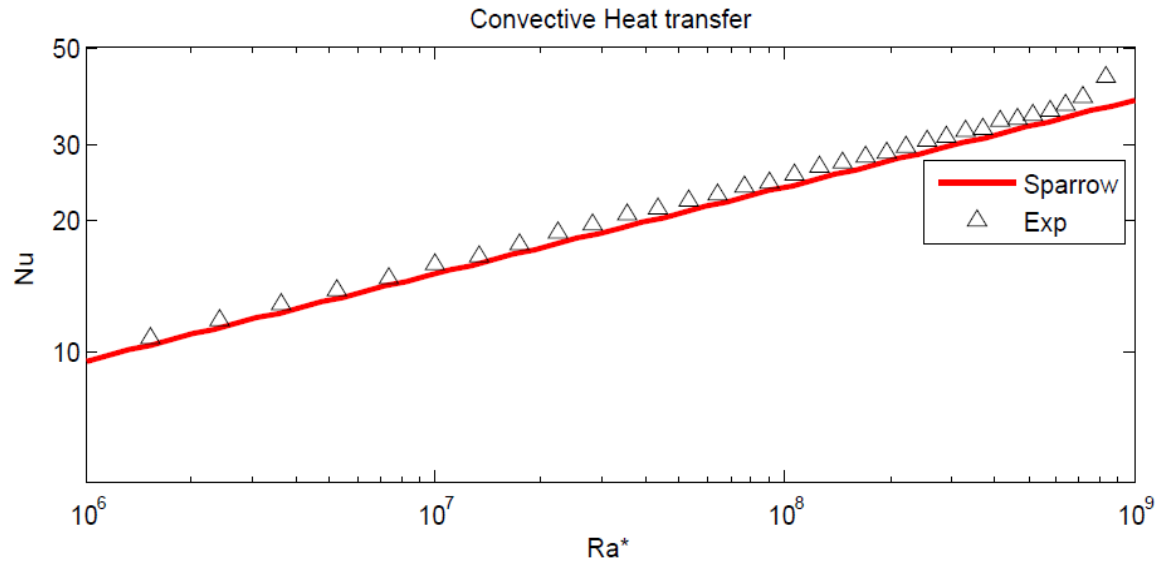

Fig. 3 Measured Nu as function of $\mathrm{Ra}^{*}$

The measurements about single row, staggered and " $V$ " type configurations are shown in figures 4,5,6; to better visualize the effect of protrusions on heat transfer and flow field, the Nu number distributions are normalized respect to $N u^{*}$.

Some qualitative considerations from the observation of contour map of fig. 4 and 5 follows:

1) the formation of a low heat transfer zone is well evident at $\alpha=0^{\circ}$ and $30^{\circ}$ (fig. $4 \mathrm{a}, 4 \mathrm{~b}, 5 \mathrm{a}, 5 \mathrm{~b}$ ) for both cases at $d=1 / 3 \mathrm{H}$ and $2 / 3 \mathrm{H}$ downstream the ribs and laterally; probably it is due to an horseshoe vortex;

2) there is the formation of a high heat transfer zone (we call it "plume" ) downstream the ribs in all cases due probably to the simultaneous action of horseshoe vortex and the tip vortex that thin the boundary layer downstream the ribs, enhancing local heat transfer;

3) The formation of a well localized peak value of $N u / N u^{*}$ excluding map at $\alpha=60^{\circ}$ and $d=2 / 3 H$ (fig. $5 d$ ); we suppose that the peak value falls outside the plate being the plume very stretched vertically; 
4) The vertical and the horizontal extent of the plume changes dramatically with the angle of attach at $d=$ $1 / 3 \mathrm{H}$ : the little plume ( about $0.6 \mathrm{~L}$ wide and $1 \mathrm{~L}$ high ) at $\alpha=0^{\circ}$ (fig. $4 \mathrm{a}$ ) grows in all directions (particularly it reaches the end of the plate ) at $\alpha=30^{\circ}$ assuming a "knife" form (fig. 4b); at $\alpha=45^{\circ}$ and $60^{\circ}$ the width of the plume decreases (fig. 4c, 4d). The behaviour at $d=2 / 3 \mathrm{H}$ is quite different: the original plume at $\alpha=$ $0^{\circ}$ (fig. $5 \mathrm{a}$ ), about $0.6 \mathrm{~L}$ wide and more than $1 \mathrm{~L}$ high, assumes a "candle flame" form (fig. $5 \mathrm{c}$ ) with a region of high value of $\mathrm{Nu} / \mathrm{Nu}^{\star}$, that is more stretched vertically at $\alpha=45^{\circ}$ respect to at $\alpha=30^{\circ}$ (respectively fig. $5 b$ and 5c).

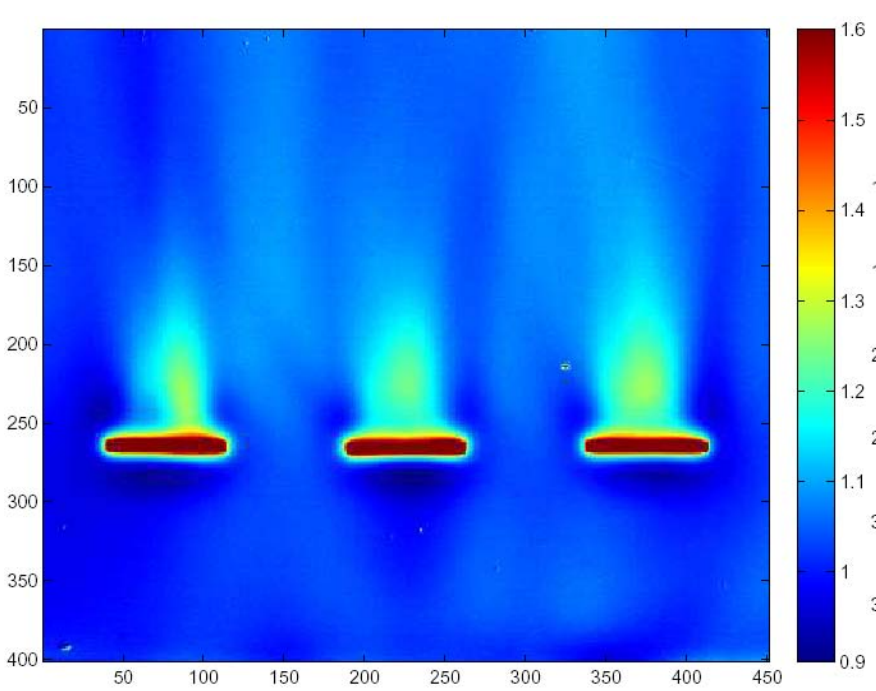

(a)

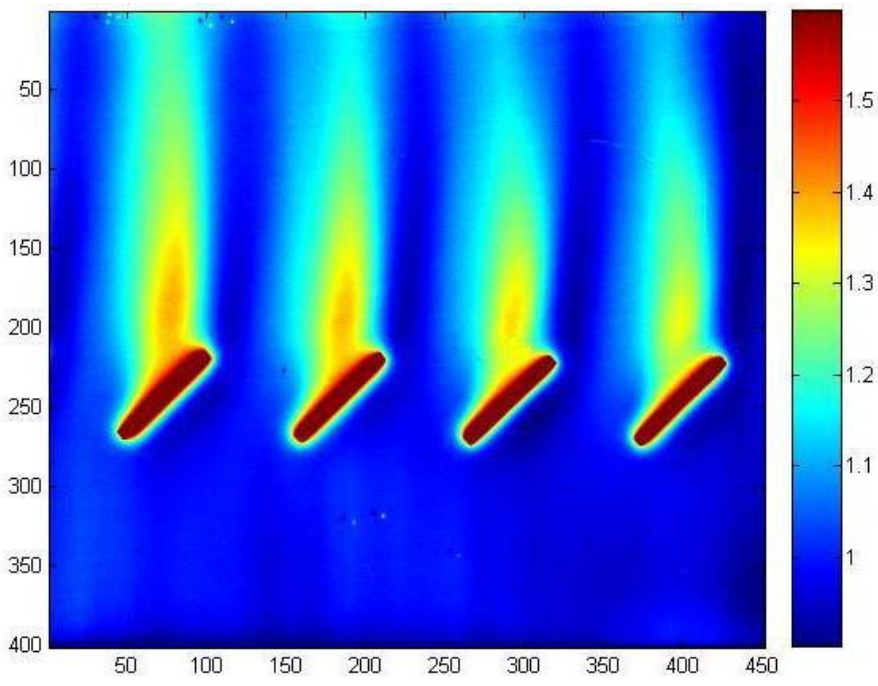

(c)

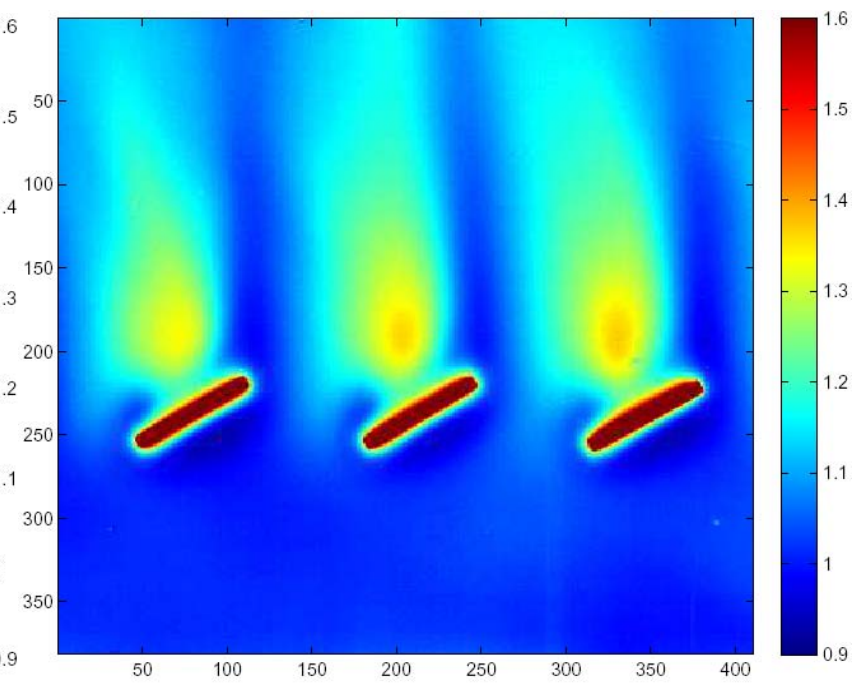

(b)

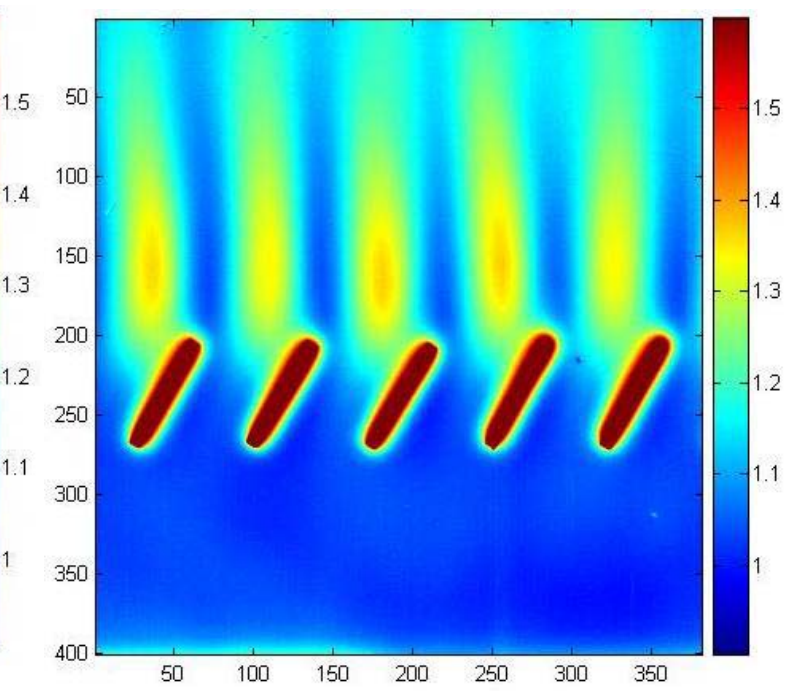

(d)

Fig. 4 Single row pattern: $\mathrm{Nu} / \mathrm{Nu}^{\star}$ map @ $d=1 / 3 \mathrm{H}$ and $\alpha=0^{\circ}(\mathrm{a}), 30^{\circ}(\mathrm{b}), 45^{\circ}(\mathrm{c}), 60^{\circ}(\mathrm{d})$. 


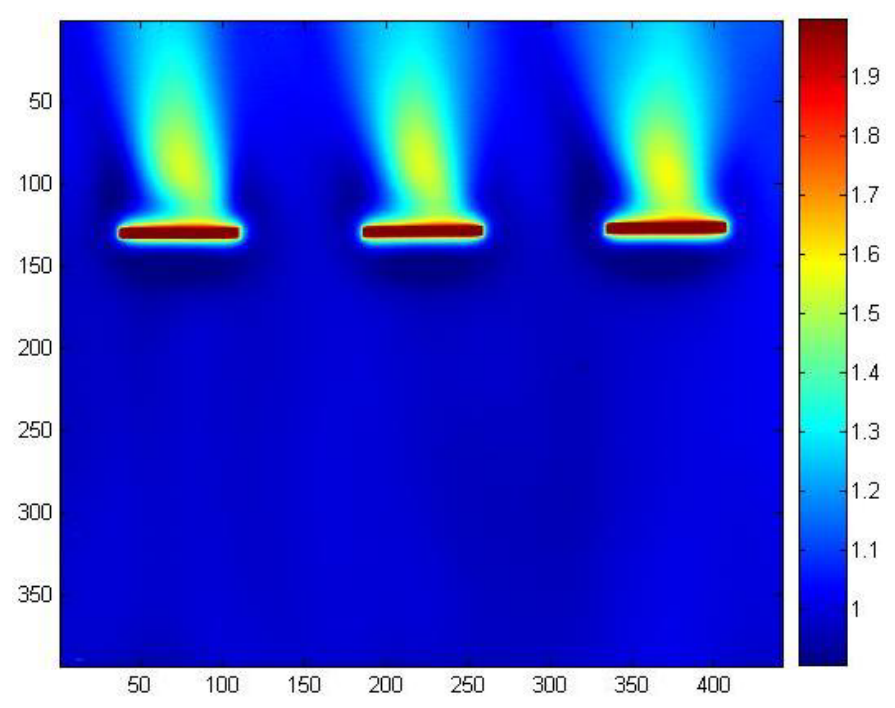

(a)

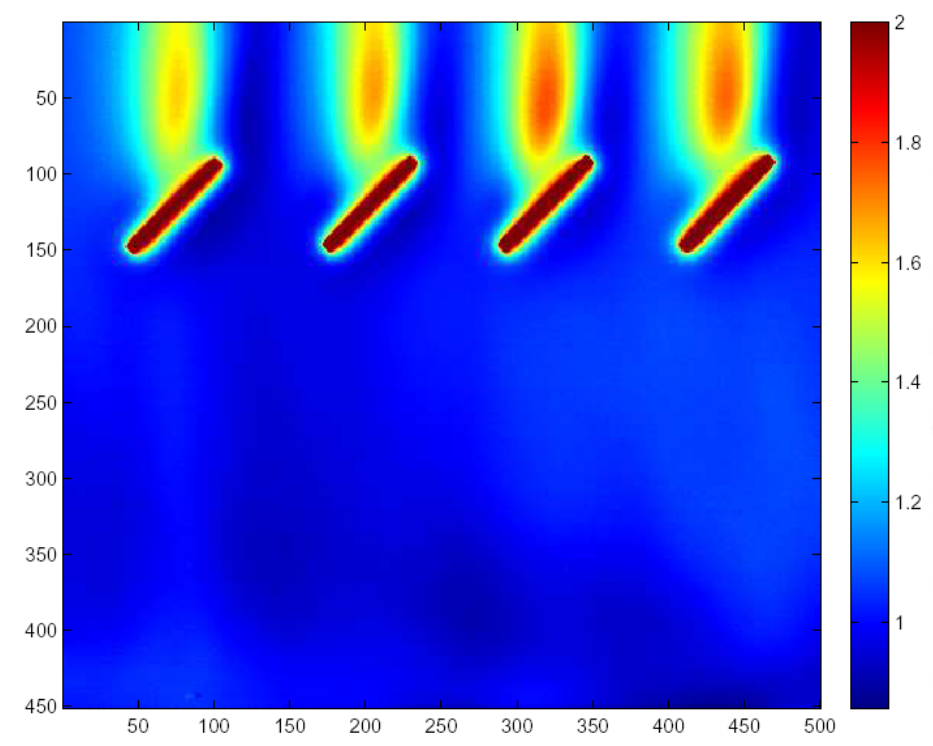

(c)

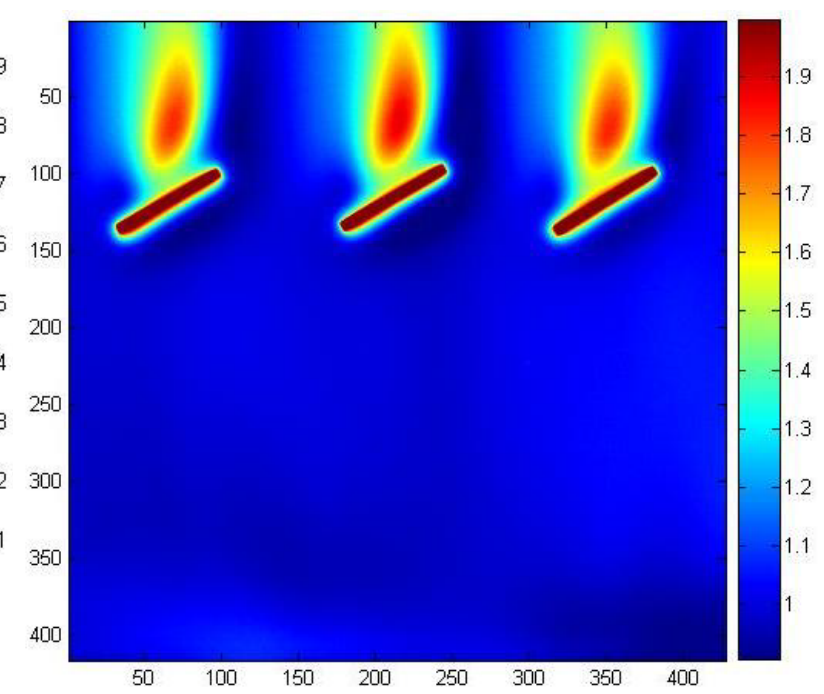

(b)

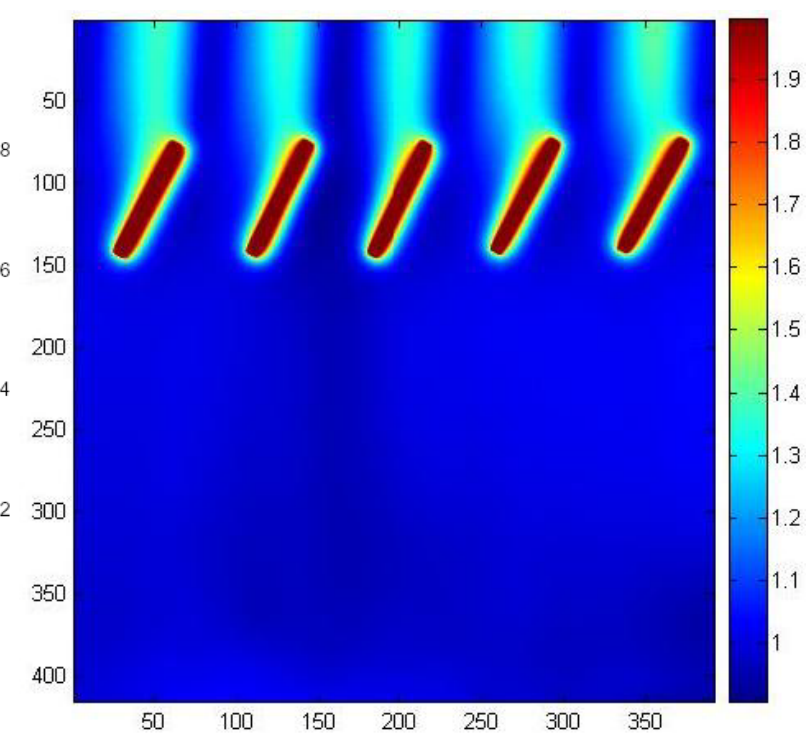

(d)

Fig. 5 Single row pattern: $\mathrm{Nu} / \mathrm{Nu} u^{*}$ map @ $d=2 / 3 \mathrm{H}$ and $\alpha=0^{\circ}(\mathrm{a}), 30^{\circ}(\mathrm{b}), 45^{\circ}(\mathrm{c}), 60^{\circ}(\mathrm{d})$.

Comparing figure $6 \mathrm{a}$ and figure $6 \mathrm{~b}$, it is clear that the interaction between two staggered rows of ribs agreed oriented with $\alpha=+45^{\circ}$ (fig. 6a) reduces dramatically the peak values of heat transfer of the top row (about $-30 \%$ ) but there is a better uniform distribution all over the plate if compared to staggered opposite orientated configuration (fig. 6b). Infact the interaction of the bottom row's plumes with the tops, although it leds to a horizontal growth of high heat transfer regions (fig. 6b) comparing with those of single row at $d=2 / 3 \mathrm{H}$ (fig. 5c), doesn't increase its peak values and additionally it decreases the local heat transfer of the lower ribs' row . $6 c$ and 6d).

Finally big regions of very high heat transfer ( $\mathrm{Nu} / \mathrm{Nu}^{*} \geq 2$ too ) are observed using "V" type ribs configurations (fig. 


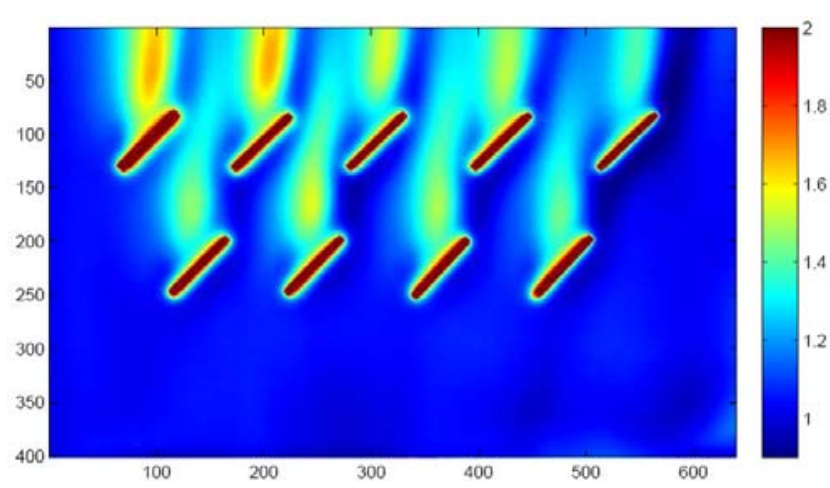

(a)

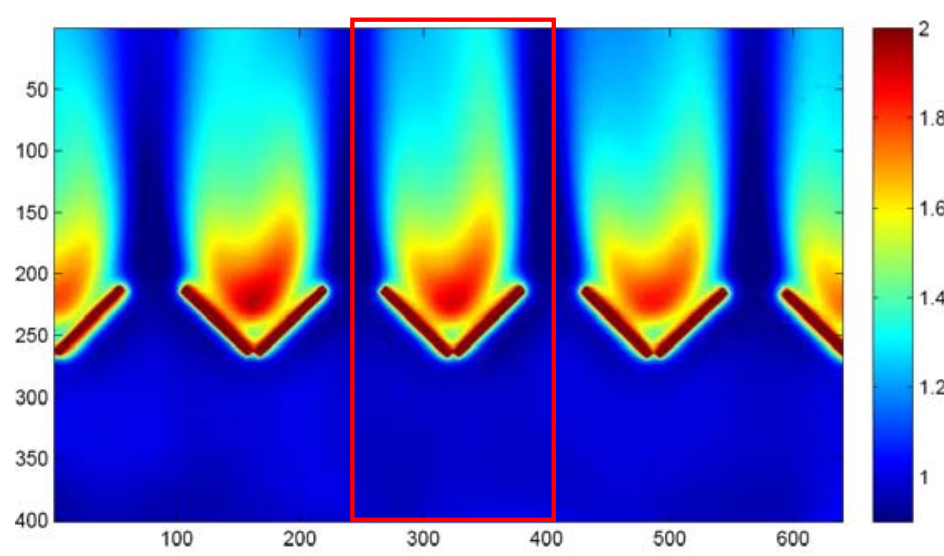

(c)

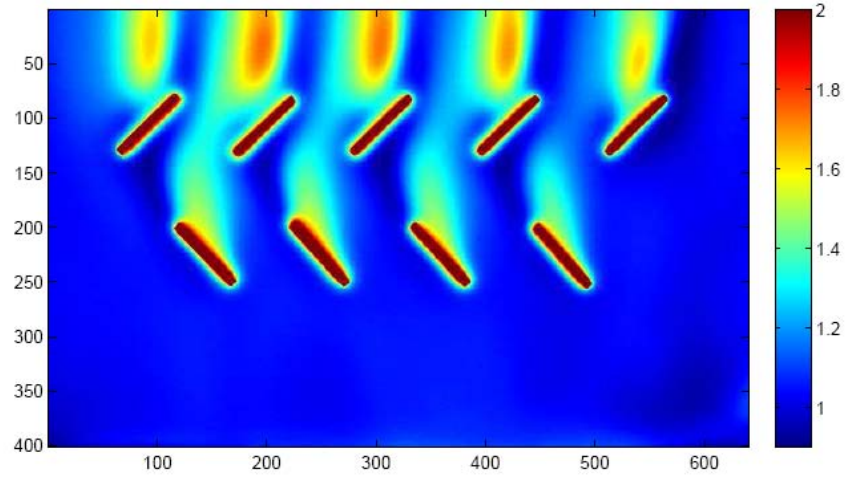

(b)

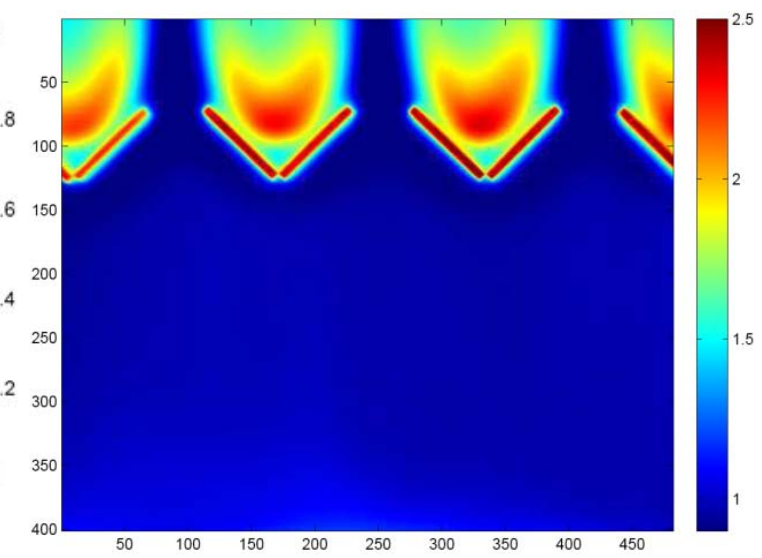

(d)

Fig. $6 \mathrm{Nu} / \mathrm{Nu} \mathrm{u}^{\star}$ map: staggered ribs with first row @ $\alpha=+45^{\circ}$ (a) and $\alpha=-45^{\circ}(\mathrm{b})$; "V" type ribs @ $d=1 / 3 \mathrm{H}$ (c), and $d=2 / 3 H(d)$. Red rectangle indicates the area used to compute mean value of $\mathrm{Nu} / \mathrm{Nu}^{*}$; values of the top face of the ribs were excluded.

Table 1 summarises the peak values and the average of $\mathrm{Nu} / \mathrm{Nu}^{*}$ of all the configurations. The last ones has been computed in the periodic area ( red rectangle in figure 6c), excluding the values on the top ribs' faces.

A maximum of enhancement of about $6 \%$ appears fixing at $d=2 / 3 \mathrm{H}$ and using single row of ribs at $\alpha=45^{\circ}$; it would seem that the configuration with $\alpha=30^{\circ}$ is the best, but an intense zone with $\mathrm{Nu} / \mathrm{Nu} * 1$ upstream opposed to its higher heat transfer zone downstream ribs is present.

A quite different behaviour is varying the angle of attack, fixing $d$ at $2 / 3 \mathrm{H}$ :there is a first local maximum (8\%) of ( $\mathrm{Nu} /$ $\left.N u^{\star}\right)_{A V E}$ at $\alpha=30^{\circ}$ and a second, more intense, value (12\%) is at $\alpha=60^{\circ}$. The "plume" of the last configuration can cover a bigger portion of periodic area (fig. 4d) than the "knife plume" (fig. 4b), enhancing average values of $N u / N u^{*}$.

The qualitative observations made previously about $\mathrm{Nu} / \mathrm{Nu} u^{*}$ map of staggered configurations are validated from calculations: staggered rows of ribs agreed oriented has a lower peak (150\% vs. 180\%) value but not much higher average value of $\mathrm{Nu} / \mathrm{Nu}(+12 \%$ vs. $+14 \%)$ respect to the opposite oriented configuration. Note that an enhancement on heat transfer of $12 \%$ and $14 \%$ can be respectively obtained also using a single row inclined of $60^{\circ}$ and "V" type configurations, both placed at $d / H=1 / 3$.

Table 1 shows also that the intensities of all heat transfer peaks of single row and also "V" type configurations at $\mathrm{d} / \mathrm{H}=2 / 3$ are higher than that at $\mathrm{d} / \mathrm{H}=1 / 3$. 
Table 1. Average values of $\mathrm{Nu} / \mathrm{Nu}^{*}$ evaluated in the periodic area (excluded ribs) and peaks of $\mathrm{Nu} / \mathrm{Nu}$.

\begin{tabular}{l|ccccc}
\hline Configuration & $d / H$ & $\alpha\left[^{\circ}\right]$ & $\left(\mathrm{Nu} / \mathrm{N} u^{\star}\right)_{\text {PEAK }}$ & $\left(\mathrm{Nu} / \mathrm{N} u^{\star}\right)_{\text {AVE }}$ & Ave. enhance [\%] \\
\hline & & 0 & 1,243 & 1,055 & 5,5 \\
& $1 / 3$ & 30 & 1,361 & 1,083 & 8,3 \\
& & 45 & 1,389 & 1,051 & 5,1 \\
\multirow{4}{*}{ Single Row } & & 60 & 1,359 & 1,123 & 12,3 \\
\cline { 2 - 6 } & & 0 & 1,550 & 1,045 & 4,5 \\
& & 30 & 1,894 & 1,046 & 4,6 \\
& $2 / 3$ & 45 & 1,770 & 1,064 & 6,4 \\
& & 60 & 1,367 & 1,018 & 1,8 \\
\hline \multirow{3}{*}{ "V" type } & $1 / 3$ & 45 & 1,909 & 1,144 & 14,4 \\
& $2 / 3$ & & 2,350 & 1,137 & 13,7 \\
\hline \multirow{3}{*}{ Staggered Ribs } & & +45 & 1,540 & 1,125 & 12,5 \\
& & -45 & 1,735 & 1,144 & 14,4 \\
\hline
\end{tabular}

\section{Conclusions and future remarks}

The paper has shown that the use of periodic pattern of ribs $(4 \mathrm{~mm} \times 11 \mathrm{~mm} \times 44 \mathrm{~mm})$ placed on a vertical flat plate in natural convection can improve convective heat transfer maximum (excluding fin effect). A few configuration of rib pattern are analysed and results are presented as $\mathrm{Nu} / \mathrm{Nu}^{*}$ maps. A good uniform distribution of surface heat transfer coefficient has been verified to be obtained using two staggered rows of ribs agreed orientated but new measurements needs since the only one inclination tested $\left(45^{\circ}\right)$. Staggered configurations of "V" type ribs could double $\left(\mathrm{Nu} / \mathrm{Nu} u^{\star}\right)_{A V E}$ of the correspondent single row value, resulting in an overall increase of $+30 \%$.

\section{Acknowledgements}

We want to thanks our graduated student, Mirengo C. for the patience during the field of experiment.

\section{REFERENCES}

[1] Bhavnani, S.H., Bergles, A.E. "Effect of surface geometry and orientation on laminar natural convection heat transfer from a vertical flat plate with transverse roughness elements". Int. J. Heat Mass Transfer 33, 965-981. 1990.

[2] Aydin, M. "Dependence of the natural convection over a vertical flat plate in the presence of the ribs" Int. Commun. Heat Mass Transfer 24, 521-531. 1997.

[3] Tanda, G. "Natural convection heat transfer in vertical channels with and without transverse square ribs" Int. J. Heat Mass Transfer 40, 2173-2185, 1997.

[4] Onbasioglu S.U., Onbastoglu H. "On enhancement of heat transfer with ribs" Applied Thermal Eng. 24. 43-57, 2004.

[5] Astarita T, Cardone G, Carlomagno GM "Infrared thermography: An optical method in heat transfer and fluid flow visualisation", Optics and Lasers In Engineering, 44 (3-4), 261-281, 2006

[6] Sparrow E.M., Gregg J.I. "Laminar free convection from a vertical plate with uniform surface heat flux" Trans. ASME 78, 435-440, 1956. 